trend for improvement between the first and second sessions. However, differences of markers of NR performance were not statistically significant.

Conclusions We detected frequent deficiencies in NR skills and no significant improvement after exposure to a single HFS NR session. In order to achieve sustained improvement in NR skills, further practice and skills-based curricula may be necessary.

\section{THE RISK LEVEL OF CANCER AMONGST ADULTS WHO WERE EXPOSED TO SECOND-HAND SMOKE AT CHILDHOOD}

doi:10.1136/archdischild-2012-302724.1543

E Odiase. Epidemiology, University of Ibadan/SmokeFree Foundation, Abuja, Nigeria

Objective To ascertain the level of risk of cancer amongst adults severely exposed to environmental tobacco smoke at child hood. Methods We conducted this study among 1,280 adults who were diagnosed for lung cancer for a period of 6 years from 2005-2011. We checked hospital records at childhood for frequent hospitalization related to tobacco effects like middle ear infections, pneumonia, bronchitis and worsened asthma conditions.

Results We were able to show the relationship between exposure to environmental tobacco smoke during childhood and cancer risk Out of the total number of participants, $2.5 \%$ percent (32 adults) had been exposed to environmental tobacco smoke (ETS) at childhood. We found that the overall cancer risk was greater for individuals with exposures to environmental tobacco smoke during both childhood and adulthood than for individuals with exposure during only one period. When specific cancer sites or types were considered, it was found that leukemia and lymphoma among adults were significantly related to exposure to maternal passive smoke before 10 years of age.

Conclusion Results of epidemiologic studies including this one provide evidence that exposure of children to environmental tobacco smoke is associated with increased rates of lower respiratory illness and increased rates of middle ear effusion, asthma, and sudden infant death syndrome. Exposure during childhood to environmental tobacco smoke may also be associated with development of cancer during adulthood.

\section{SOCIOECONOMIC STATUS LOWER LEVELS OF PARENTAL KNOWLEDGE ABOUT CHILD ABUSE, NEGLECT, EXPERIENCES AND DISCIPLINE METHODS USED}

doi:10.1136/archdischild-2012-302724.1544

'Y Dallar Bilge, 'MA Taşar, 'B Kılınçoğlu, 'S Ozmen, 2Ü Tıraş. 'Paediatrics, Ankara Training and Research Hospital, Ankara; ${ }^{2}$ Neonatology, Acıbadem Hospital, Istanbul, Turkey

Aim The purpose of this study is families with low socioeconomic status to determine level of knowledge about child abuse and neglect, experiences, disciplinary methods used by children of families and the factors affecting them.

Materials and Methods Children's Clinic admitted that the level of income below the poverty line in 1043 was the child's parent survey. Sociodemographic characteristics, parents' level of knowledge about child abuse-neglect, and about their own childhood experiences, their thoughts and behaviors were recorded in the survey.

Results $17.7 \%$ of the parents' have received information about child abuse-neglect, $43.2 \%$ has suffered neglect, and $37.6 \%$ has suffered abuse. We asked "How do you watch on an attitude of an experienced in the case of abuse" $68.6 \%$ of parents said "consult official institutions', $31.4 \%$ of parents said 'family should be deal with a problem or saved within family'. $68.5 \%$ of the parents' said does not threaten, $22.3 \%$ of families said threatened emotionally then $2.0 \%$ of families said threatened with physical punishment.
With the increase of education level of parents an increase in the rate of parents who said 'I don't threatened'. We asked to parents 'How to punish your children?' $47.0 \%$ of families said not to punish.

Conclusion The majority of neglect and abuse suffered by the parents and they think that they deserve punishment was the same group of children. Emotional and physical abuse in our society children are still being used as an education and disciplinary methods.

\section{THE USE OF DRUG MANIPULATION TO OBTAIN DOSES REQUIRED IN PAEDIATRIC PRACTICE: A SYSTEMATIC REVIEW}

doi:10.1136/archdischild-2012-302724.1545

${ }^{1}$ RH Richey, ${ }^{2} \mathrm{JV}$ Craig, ${ }^{1,3} \mathrm{UU}$ Shah, ${ }^{4} \mathrm{JL}$ Ford, ${ }^{1} \mathrm{CE}$ Barker, ${ }^{1,3} \mathrm{M}$ Peak, ${ }^{1} \mathrm{AJ}$ Nunn, ${ }^{5,6} \mathrm{MA}$ Turner. 'AlderHey Children's NHS Foundation Trust, Liverpool; '2Faculty of Medicine and Health Sciences, University of East Anglia, Norwich; ${ }^{3}$ Cheshire, Merseyside \& North UK Medicines for Children Local Research Network; 'Liverpool John Moores University; ${ }^{5}$ Liverpool Women's NHS Foundation Trust; ${ }^{6}$ NIHR Medicines for Children Research Network Co-ordinating Centre, Liverpool, UK

Background and Aims To determine whether there is an evidence base for drug manipulation to obtain the required dose, a common feature of paediatric clinical practice.

Methods Systematic review, including studies that considered the dose accuracy of manipulated medicines of any dosage form, evidence of safety or harm, bioavailability, patient experience, tolerability, contamination and comparison of methods of manipulation. Results 43 studies were eligible for inclusion, 42 of which involved tablets being cut, split, crushed or dispersed. The remaining one study involved the manipulation of suppositories of one drug. No eligible studies concerning manipulation of oral capsules or liquids, rectal enemas, nebuliser solutions, injections or transdermal patches were identified. Seventeen of the tablet studies considered dose accuracy using weight and/or drug content. In studies that considered weight using adapted pharmacopoeial specifications, the percentage of halved tablets meeting these specifications ranged from $33 \%$ to $93 \%$. Nine studies investigated bioavailability outcomes following the manipulations of five delayed release formulations. In all nine studies all of the dosage form was administered. Only one study was identified where drugs were manipulated to obtain a proportion of the dosage form, and that proportion administered. The eight studies that considered patient experience found that having to manipulate the tablets did not have a negative impact on adherence. Of the 43 studies only two studies reported investigating children.

Conclusion This review yielded limited evidence to support manipulation of medicines. The results cannot be extrapolated between dosage forms, methods of manipulation or between different brands of preparation.

\section{CONGENITAL HYPOTHYROIDISM: A REVIEW OF THE RISK FACTORS}

doi:10.1136/archdischild-2012-302724.1546

'S Dalili, ${ }^{1,2}$ SM Rezvany, ${ }^{1} \mathrm{~A}$ Dadashy, ${ }^{3} \mathrm{H}$ Mohamady, ${ }^{4} \mathrm{H}$ Dalili, ${ }^{5} \mathrm{~A}$ Medghalchy, ${ }^{1} \mathrm{H}$ Gholam Nezhad, 'M Mirza Nezhad, 'H Rokhshad. 'Vice- Chancellor of Health ${ }^{2}$ Health Care Center, Guilan University of Medical Sciences; ${ }^{3}$ Pediatric, Shiraz University of Medical Sciences; " ${ }^{B}$ Breast Feeding Center, Tehran University of Medical Sciences; ${ }^{5}$ Ophtalmology, Guilan University of Medical Sciences, Rasht, Iran

Objective Higher prevalence of $\mathrm{CH}$ among Iranian population necessitates review of risk factors and distribution of these factors among whole populations.

Method In Guilan province during years 2006 to 2010, neonatal screening for TSH was measured in 3-5 days after birth. All neonate with TSH level $>=5 \mathrm{mu} / \mathrm{l}$ refer to endocrinologist and serum TSH, 